\title{
$N$-dimensional alternate coined quantum walks from a dispersion-relation perspective
}

\author{
Eugenio Roldán, ${ }^{1}$ Carlo Di Franco, ${ }^{2,3}$ Fernando Silva, ${ }^{1}$ and Germán J. de Valcárcel ${ }^{1}$ \\ ${ }^{1}$ Departament d'Òptica, Universitat de València, Dr. Moliner 50, 46100-Burjassot, Spain \\ ${ }^{2}$ Departamento de Química Física, Universidad del País Vasco-Euskal Herriko Unibertsitatea, Apartado 644, E-48080 Bilbao, Spain \\ ${ }^{3}$ CTAMOP, School of Mathematics and Physics, Queen's University, Belfast BT7 1NN, United Kingdom
}

(Received 13 July 2012; published 25 February 2013)

\begin{abstract}
We suggest an alternative definition of $N$-dimensional coined quantum walk by generalizing a recent proposal [Di Franco et al., Phys. Rev. Lett. 106, 080502 (2011)]. This $N$-dimensional alternate quantum walk, AQW $(N)$, in contrast with the standard definition of the $N$-dimensional quantum walk, $\mathrm{QW}_{(N)}$, requires only a coin qubit. We discuss the quantum diffusion properties of $\mathrm{AQW}_{(2)}$ and $\mathrm{AQW}_{(3)}$ by analyzing their dispersion relations that reveal, in particular, the existence of diabolical points. This allows us to highlight interesting similarities with other well-known physical phenomena. We also demonstrate that $\mathrm{AQW}_{(3)}$ generates considerable genuine multipartite entanglement. Finally, we discuss the implementability of AQW $(N)$.
\end{abstract}

DOI: 10.1103/PhysRevA.87.022336

PACS number(s): 03.67.Ac, 03.67.Bg, 05.40.Fb

In both its standard forms, the coined [1] and the continuous one [2], quantum walk is the quantum version of a classical random process, described by the diffusion and the telegrapher's equations, respectively [3]. In the coined quantum walk - the process we consider here-there is a system (the walker) that undergoes a conditional displacement, to the right or the left, depending on the output of a coin throw, as in the random walk. But differently from its classical counterpart, here both coin and walker are quantum in nature. The onedimensional coined quantum walk- $\mathrm{QW}_{(1)}$ for short-has been studied from many different perspectives, especially from the quantum computational point of view [4]. In the last few years, quantum walks have also received increasing experimental attention [5-7], including cases with more than one particle [8].

The situation is quite different when dealing with $\mathrm{N}$ dimensional quantum walks, $\mathrm{QW}_{(N)}$ for short. They were first discussed by Mackay et al., who introduced them in complete analogy with $\mathrm{QW}_{(1)}$ [9] (see also Ref. [10]). As defined in Ref. [9], $\mathrm{QW}_{(N)}$ requires the use of a $2^{N}$-dimensional qudit as coin, as well as a coin operator represented by a $2^{N} \times 2^{N}$ unitary matrix. This introduces increasing complexity in the process as $N$ grows, especially from the experimental viewpoint $[11,12]$, but also from the theoretical one $[13,14]$. However, Di Franco et al. [15] have recently proposed an alternative twodimensional quantum walk, namely, the alternate quantum walk-AQW for short-that is simpler than the standard one. In $\mathrm{AQW}$ the coin is a single qubit, as in $\mathrm{QW}_{(1)}$, and each time step is divided into two halves: in the first one the coin is thrown (i.e., a Hadamard transformation is applied on the coin qubit) and the conditional displacement along $x$ is performed; then, in the second half of the time step, the coin is thrown again and the conditional displacement along $y$ is performed. Hence, in $\mathrm{AQW}$, the four-dimensional qudit of $\mathrm{QW}_{(2)}$ is replaced by a single qubit, the price paid for that being to double the number of substeps per single time step. Quite unexpectedly, AQW reproduces the same spatial probability distributions of $\mathrm{QW}_{(2)}$ when the Grover coin is used - Grover-QW $\mathrm{Q}_{(2)}$ for short - for a set of particular initial conditions, precisely those for which the characteristic localization of Grover-QW $(2)$ does not occur. In Refs. [15,16] analytical demonstrations of the (partial) equivalence between Grover- $\mathrm{QW}(2)$ and $\mathrm{AQW}$ are given.

Here we generalize AQW to $N$ dimensions: we define the $N$-dimensional alternate coined quantum walk - $\mathrm{AQW}_{(N)}$ for short - as a quantum walk in which the time steps are divided into $N$ substeps. In each of these substeps, the coin throw is followed by the conditional displacement along one of the $N$ dimensions. In a single time step of $\mathrm{AQW}_{(N)}$, the qubit coin is therefore thrown $N$ times, but this is clearly simpler than throwing a single $2^{N}$-dimensional coin, from both the experimental and analytical points of view, in particular for large $N$ (a more detailed discussion about the possible scaling of errors strongly depends on the physical setting exploited for the realization of the scheme). We show below that, besides a simpler experimental implementability as compared with $\mathrm{QW}_{(N)}, \mathrm{AQW}_{(N)}$ has a rich dynamics that is also easy to understand. We provide some analytical results concerning the evolution of the probability distribution for cases $N=2,3$, paying special attention to the processes' dispersion relations. These reveal the existence of diabolical points (DPs in the following), conical intersections involving a degeneracy [17], that allow us to highlight interesting similarities with other well-known physical phenomena. As a striking example, we present the homogeneous propagation of an initially extended state with perfect circular symmetry in relation to the ring shape of the beam exiting a biaxial crystal under conical refraction conditions [18]. Further physical contexts where DPs appear are given in the remainder of the paper. An analysis from this viewpoint has not been performed so far and is useful to broaden our knowledge of the particular scheme under investigation. We also demonstrate the generation of considerable genuine multipartite entanglement in $\mathrm{AQW}_{(3)}$. We conclude this paper with a brief discussion concerning the experimental implementability of $\mathrm{AQW}_{(N)}$.

\section{THE MODEL}

In order to introduce $\mathrm{AQW}_{(N)}$ formally, let $|\psi\rangle_{t}$ represent the state of the system at (discrete) time $t$. The vector $|\psi\rangle_{t}$ is defined in the compound Hilbert space $\mathcal{H}_{P} \otimes \mathcal{H}_{C}$, where $\mathcal{H}_{P}$ and $\mathcal{H}_{C}$ are the Hilbert spaces for the lattice sites and coin qubit, respectively. With $\mathcal{H}_{P}$ and $\mathcal{H}_{C}$ spanned by $\left\{|\vec{x}\rangle, \vec{x} \in \mathbb{Z}^{N}\right\}$ 
and $\{|c\rangle, c=u, d\}$, we can write $|\psi\rangle_{t}=\sum_{\vec{x}} \sum_{c=u, d} c_{\vec{x}, t}|\vec{x} ; c\rangle$ with $|\vec{x} ; c\rangle=|\vec{x}\rangle \otimes|c\rangle$, where $c_{\vec{x}, t}$ is the probability amplitude for the walker to be at site $\vec{x}=\left(x_{1}, \ldots, x_{N}\right)$ at time $t$ with the coin in state $c$. The probability of finding the walker at site $\vec{x}$ at time $t$ is $P_{\vec{x}, t}=\left|u_{\vec{x}, t}\right|^{2}+\left|d_{\vec{x}, t}\right|^{2}$.

The state evolves as $|\psi\rangle_{t+1}=\hat{U}_{(N)}|\psi\rangle_{t}$, with $\hat{U}_{(N)}=$ $\hat{D}_{N} \hat{C}_{N} \hat{D}_{N-1} \hat{C}_{N-1} \ldots \hat{D}_{1} \hat{C}_{1}$ a unitary operator. Here, operator $\hat{C}_{i}$ is the coin operator, acting only in $\mathcal{H}_{C}$, whose more general form is $\hat{C}_{i}=\cos \theta_{i}\left(|u\rangle\left\langle u\left|-e^{i\left(\alpha_{i}+\beta_{i}\right)}\right| d\right\rangle\langle d|\right)+$ $\sin \theta_{i}\left(e^{i \alpha_{i}}|u\rangle\left\langle d\left|+e^{i \beta_{i}}\right| d\right\rangle\langle u|\right)$, with $\left(\alpha_{i}, \beta_{i}, \theta_{i}\right)$ arbitrary reals and $i=1, \ldots, N$ (notice that, for $\alpha_{i}=\beta_{i}=0$ and $\theta_{i}=\pi / 4$, $\hat{C}_{i}$ is just the Hadamard transformation). $\hat{D}_{i}$ is the conditional displacement operator along direction $x_{i}$, which we write as $\hat{D}_{i}=\sum_{\vec{x} \in \mathbb{Z}^{N}}\left[\left|\vec{x}+\vec{n}_{i} ; u\right\rangle\left\langle\vec{x} ; u|+| \vec{x}-\vec{n}_{i} ; d\right\rangle\langle\vec{x} ; d|\right]$, where $\vec{n}_{i}$ is the unit vector along direction $x_{i}$.

Equation $|\psi\rangle_{t+1}=\hat{U}_{(N)}|\psi\rangle_{t}$ can be expressed as a map relating probability amplitudes $c_{\vec{x}, t+1}$ with $\tilde{c}_{\vec{x}^{\prime}, t}$, where $c, \tilde{c}=$ $u, d$ and $\vec{x}^{\prime}$ are nearest neighbors of $\vec{x}$. This map admits two plane-wave solutions of the form $\operatorname{col}\left(u_{\vec{x}, t}, d_{\vec{x}, t}\right)_{ \pm}=$ $\vec{\varphi}_{\vec{q}, \pm} \exp \left[i\left(\vec{q} \cdot \vec{x}-\omega^{( \pm)} t\right)\right]$, where $\vec{\varphi}_{\vec{q}, \pm}=\operatorname{col}\left(u_{\vec{q}, \pm}, d_{\vec{q}, \pm}\right)$ are time-independent vectors, $\vec{q}$ is the pseudomomentum with $q_{i} \in(-\pi, \pi]$, and $\omega^{( \pm)}$are two frequencies determined by the dispersion relation. The dispersion relation is most relevant because $|\psi\rangle_{t}$ is entirely determined by it, given $|\psi\rangle_{t=0}$. Moreover, when the initial state extends over a finite set of points in the lattice, especially when it is modulated by a smooth function of space, the dispersion relation is particularly useful for predicting the evolution of the initial wave packet, due to the relatively well-defined group velocity (given by the local gradient of the dispersion-relation curve). In this case, long-wavelength continuous approximations are very well suited and useful for envisaging the long time behavior of the probability distribution. This has been discussed in detail for $\mathrm{QW}_{(1)}$ in Ref. [19] (see also Refs. [3,20]). Here, we will limit ourselves to a qualitative discussion of what the dispersion relation suggests for cases $N=2,3$.

\section{DISPERSION RELATION FOR TWO-DIMENSIONAL ALTERNATE QUANTUM WALK}

Let us first consider $\mathrm{AQW}_{(2)}$. By proceeding as stated above, one obtains the following dispersion relation:

$$
\cos \Omega=c_{1} c_{2} \cos (u+v)+s_{1} s_{2} \cos (u-v),
$$

where $c_{i}=\cos \theta_{i}, \quad s_{i}=\sin \theta_{i}, \quad u=q_{1}+\left(\beta_{1}+\alpha_{2}\right) / 2, \quad v=$ $q_{2}+\beta_{2} / 2$, and $\Omega=\omega-\left(\beta_{1}+\beta_{2}+\alpha_{2}\right) / 2$. Notice that phase $\alpha_{1}$ does not appear in Eq. (1); hence it is irrelevant. As for the phases that do appear, they just entail a translation of the frequency and spatial quasimomentum. Only $\theta_{1}$ and $\theta_{2}$ are dynamically relevant parameters. Figure 1 (a) presents the dispersion curves for $\theta_{1}=\theta_{2}=\pi / 4$. The most relevant features are the existence of a number of saddle points (for which the group velocity is zero), together with regions of maximum slope (which equals 0.5 , hence the maximum velocity in $\mathrm{AQW}_{(2)}$ ) and, most importantly, the existence of DPs. Interestingly, when $\theta_{1} \neq \theta_{2}$, the DPs disappear, as shown in Fig. 1(b). This suggests that the existence of DPs could be particularly sensitive to decoherence effects in the coin mechanism. (a)

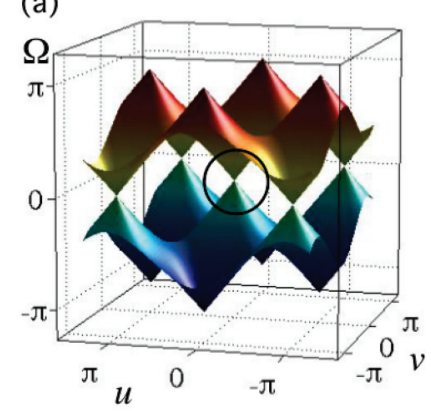

(c)

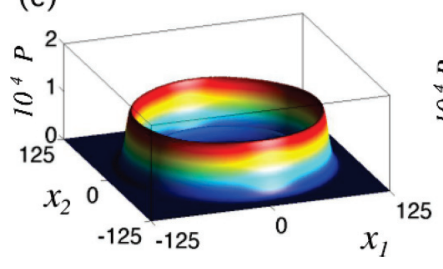

(b)

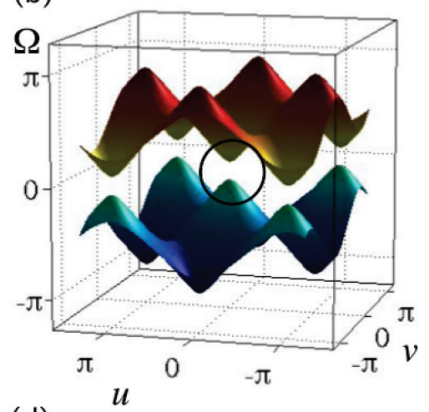

(d)

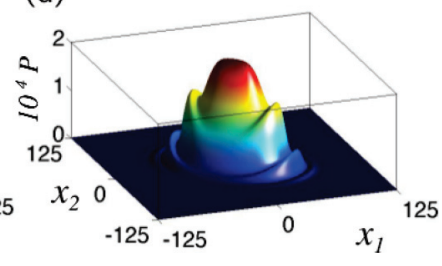

FIG. 1. (Color online) 3D views of the two branches of Eq. (1), for $\theta_{1}=\theta_{2}=\pi / 4$ (a) and $\theta_{1}=\pi / 4 \neq \theta_{2}=\pi / 3$ (b). Propagation in $\mathrm{AQW}_{(2)}$ after 90 time steps of an initial state with a Gaussian probability distribution of width $\sigma_{H W H M}=7$ and coin state $\operatorname{col}(1 / \sqrt{2}, i / \sqrt{2})$ equal for all populated sites, for $\theta_{1}=\theta_{2}=\pi / 4$ (c) and $\theta_{1}=\pi / 4 \neq \theta_{2}=\pi / 3$ (d).

The dynamics around the points of null and maximum slope can be analyzed as in Ref. [19] for $\mathrm{QW}_{(1)}$, i.e., in terms of known solutions of simple linear wave equations. One can then envisage more or less straightforward generalizations of the results there discussed to the two-dimensional case (we study this elsewhere for Grover-QW $\mathrm{QW}_{(2)}$ [21]). But the existence of DPs is particularly appealing and constitutes a qualitative difference with the one-dimensional case. This geometric object, the DP (that takes its name from the diabolo-like shape of the conical intersection), appears in physics in quite different contexts such as, for instance, quantum triangular billiards [17], conical refraction in crystal optics [18], the electronic spectrum of polyatomic molecules [22], or the dispersion relations for massless fermions (Dirac electrons) in QED and for electrons in graphene [23,24] or optical lattices [25]. The diabolo is associated with some remarkable phenomena appearing in those systems. As for a given initial condition the dispersion relation determines the evolution of the system (in the absence of dissipation), the existence of DPs establishes a strong link between the evolution properties in these contexts. Let us remark the fact that the quantum walk, differently from the continuous systems mentioned above, is defined in a discrete Hilbert space.

For what we have stated so far, we can expect to find in the dynamics of $\mathrm{AQW}_{(2)}$ some parallelism with phenomena present in the aforementioned systems. In this sense, Fig. 1(c) shows the propagation of an initially extended state [with a Gaussian probability distribution of $\sigma_{H W H M}=7$ and coin state $\operatorname{col}(1, i) / \sqrt{2}$ equal for all populated sites] after 90 time steps. A homogeneous ballistic propagation from the origin with perfect circular symmetry is clearly visible, which strongly recalls the ring shape of the beam exiting a biaxial crystal under conical refraction conditions [18]. Indeed, a careful analysis reveals that the fine structure of the probability distribution in 
Fig. 1(c) is very similar to that appearing in conical refraction (the so-called Pogendorf rings [18]), a result to be reported elsewhere with full mathematical details [21]. It is interesting to compare this dynamics with that shown in Fig. 1(d), which has been obtained for the same initial conditions but with $\theta_{1}=\pi / 4 \neq \theta_{2}=\pi / 3$. In this case the diabolo is lost and the branches of the dispersion relation show a parabolic shape [see Fig. 1(b)] that leads to evolutions typical of linear optical diffraction [19]. We want to mention that a similar controlled disappearance of the DP has been experimentally observed in graphene [23].

Before moving to a higher dimension, let us revisit the relation between $\mathrm{AQW}_{(2)}$ and Grover- $\mathrm{QW}_{(2)}$ from the dispersion-relation perspective. The dispersion relation for Grover-QW $\mathrm{W}_{(2)}$ consists of four sheets, because the coin space is four-dimensional, and can be found in Refs. [14,21]. In our notation, they read $\omega_{1,2}=0, \pi$ and $\omega_{3,4}= \pm \arccos [(\cos u+$ $\cos v) / 2$ ]. Remarkably, the two sheets $\omega_{3,4}$ coincide with those of $\mathrm{AQW}_{(2)}$ [Eq. (1)] for $\theta_{1}=\theta_{2}=\pi / 4$ after identifying $(u, v)$ in Grover-QW ${ }_{(2)}$ with $(u+v, u-v)$ in $\mathrm{AQW}_{(2)}$, i.e., the two dispersion relations coincide for these parameters up to a $\pi / 4$ rotation of the pseudomomentum. The two other roots in Grover-QW $\mathrm{W}_{(2)}, \omega_{1,2}=0, \pi$, are constant, which means that the projections of the initial state onto the corresponding eigenvectors will not evolve in time. This is the origin of localization in Grover-QW $\mathrm{W}_{(2)}$ for most initial coin states, as already noticed in Ref. [14]. We conclude that whenever the initial state in Grover-QW $\mathrm{Q}_{(2)}$ does not project onto the eigenvectors governed by $\omega_{1,2}$, Grover-QW $(2)$ and $\mathrm{AQW}_{(2)}$ are isomorphous for $\theta_{1}=\theta_{2}=\pi / 4$. This is our proof of the (partial) equivalence between the two versions of the process.

\section{DISPERSION RELATION FOR THREE-DIMENSIONAL ALTERNATE QUANTUM WALK}

Let us now move to $\mathrm{AQW}_{(3)}$. The dispersion relation is governed by

$$
\begin{aligned}
\sin \Omega= & c_{1}\left[c_{2} c_{3} \sin (u+v+w)+s_{2} s_{3} \sin (u-v+w)\right] \\
& +s_{1}\left[c_{2} s_{3} \sin (u+v-w)-s_{2} c_{3} \sin (u-v-w)\right],
\end{aligned}
$$

with $c_{i}=\cos \theta_{i}, s_{i}=\sin \theta_{i}$, and $(u, v, w, \Omega)=\left(q_{1}, q_{2}, q_{3}, \omega\right)+$ $\left(\delta q_{1}, \delta q_{2}, \delta q_{3}, \delta \omega\right)$. Here $2 \delta q_{1}=\alpha_{1}+\beta_{2}, 2 \delta q_{2}=\alpha_{2}+\beta_{3}$, $2 \delta q_{3}=\alpha_{3}+\beta_{1}$, and $\delta \omega=-\left(\delta q_{1}+\delta q_{2}+\delta q_{3}\right)$. From Eq. (2) two dispersion relations are obtained, namely, $\omega^{(+)}=\Omega$ and $\omega^{(-)}=\pi-\omega^{(+)}$. As in $\mathrm{AQW}_{(2)}$, some phases in $\hat{C}_{i}$ are absent in the dispersion relation (hence they are irrelevant) and the effect of the rest of phases in $\hat{C}_{i}$ is just a displacement of the dispersion-relation surfaces in the $\left\{q_{1}, q_{2}, q_{3}, \omega\right\}$ space. Equation (2) is simpler for $\theta_{i}=$ $\pi / 4, i=1,2,3$. In this case there are eight degeneracies (occurring when $\left.\omega^{(+)}=\omega^{(-)}=\pi / 2\right)$ at $\left(u_{\mathrm{DP}}, v_{\mathrm{DP}}, w_{\mathrm{DP}}\right)=$ $\{(a, a, a),(-a,-a, b),(-a, b,-a),(b,-a,-a)\}$, with $a, b=$ $\pi / 4,3 \pi / 4$, and $a \neq b$. These are the three-dimensional (3D) equivalents of the DPs discussed for $\mathrm{AQW}_{(2)}$. As in $\mathrm{AQW}_{(2)}$, the DPs disappear when phases $\theta_{i}$ are different. We mention that several crystallographic structures have recently been proposed for obtaining 3D DPs (Dirac-semimetal in 3D) [26]. (a)

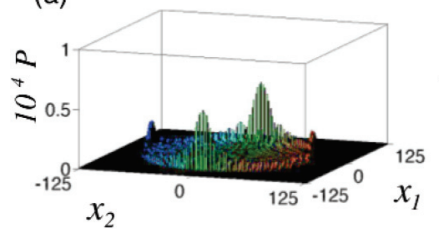

(c)

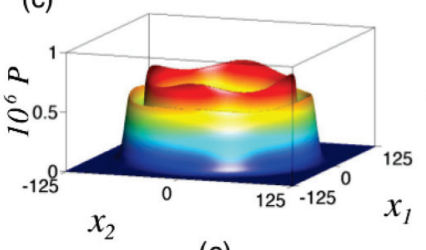

(e)

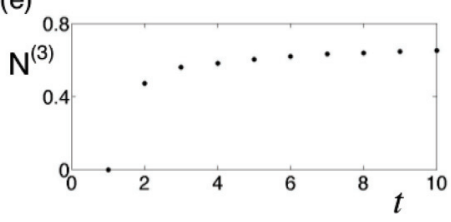

FIG. 2. (Color online) Propagation in $\mathrm{AQW}_{(3)}$ after 90 time steps of $(\mathrm{a}, \mathrm{b})$ a localized initial state and $(\mathrm{c}, \mathrm{d})$ a spatially extended initial state with Gaussian probability distribution of width $\sigma_{H W H M}=$ 7, whose pseudomomentum is centered at one of the DPs $\left[\left(u_{\mathrm{DP}}, v_{\mathrm{DP}}, w_{\mathrm{DP}}\right)=(\pi / 4, \pi / 4,-3 \pi / 4)\right]$. In both cases $\theta_{1}=\theta_{2}=\theta_{3}=$ $\pi / 4$ and the initial coin state is $\operatorname{col}(0,1)$. Panel (e) shows the tripartite negativity $N^{(3)}$ against the number of time steps $t$ in $\mathrm{AQW}_{(3)}$, with the walker starting at the origin and initial coin state $\operatorname{col}(1, i) / \sqrt{2}$.

In Figs. 2(a) and 2(b) we present two bidimensional projections of the probability distribution corresponding to the propagation of a walker initially localized at the origin. The width of the distribution grows linearly with time, along all three spatial dimensions, as it happens for lower dimensionality. In order to show the effect of the DP, Figs. 2(c) and 2(d) show the same projections as Figs. 2(a) and 2(b) when the initial condition of the walker is not localized but extended. Again we choose a Gaussian distribution with $\sigma_{H W H M}=7$, whose pseudomomentum is centered at one of the DPs $\left[\left(u_{\mathrm{DP}}, v_{\mathrm{DP}}, w_{\mathrm{DP}}\right)=(\pi / 4, \pi / 4,-3 \pi / 4)\right]$. We observe a symmetric ballistic dynamics in the $\left(x_{1}, x_{2}\right)$ plane [Fig. 2(c)] that resembles that of Fig. 1(c). Notice the existence of two concentric bright rings, as it occurs in conical refraction [17]. However, the probability is not equally symmetric in the $\left(x_{1}, x_{3}\right)$ plane [Fig. 2(d)], which reveals an intrinsic lack of symmetry in $\mathrm{AQW}_{(3)}$ : indeed, it can be shown that it is not possible to find any initial coin state that leads to a symmetric propagation in all directions [the chosen initial coin state, $\operatorname{col}(0,1)$ for all populated sites, leads to a symmetric distribution in the planes with constant $x_{3}$, but not in those for which $x_{3}$ varies, as the figures show]. One could wonder whether $\mathrm{AQW}_{(3)}$ is a process similar to Grover-QW $\mathrm{QW}_{(3)}$ as it happens for $N=2$. The answer is negative: we have derived and compared the dispersion relations of both processes for $N=3$ and they are different. Hence $N=2$ is a singular case in this respect.

\section{GENERATION OF MULTIPARTITE ENTANGLEMENT}

Another aspect investigated in the context of twodimensional quantum walks is the generation and the effects of bipartite entanglement during their evolution [27]. Having 
a richer and more complex structure than the bipartite case [28], multipartite entanglement has recently attracted a lot of interest in the scientific community. Clearly, a feasible system able to generate a proper amount of genuine multipartite entanglement could be a valuable benchmark for this rapidly growing research field. Hence a most relevant question is whether quantum walks with $N>2$ do exhibit considerable genuine multipartite entanglement or not. We have investigated this for $N=3$. We have first to trace out the state of the coin, and we are then left with a density matrix in the composite Hilbert space $\mathcal{H}_{x_{1}} \otimes \mathcal{H}_{x_{2}} \otimes \mathcal{H}_{x_{3}}$ (each subspace corresponding to a direction of the walk). We evaluate the multipartite entanglement present in this composite system by means of the tripartite negativity [29]. This is defined as the geometric average of the three negativities that are obtained by considering the three possible bipartitions of the total system, giving $N^{(3)}=\sqrt[3]{N_{1-23} N_{2-13} N_{3-12}}$. Here $N_{i-j k}$ is the negativity of the composite system $\{i, j, k\}$ corresponding to the bipartition in the subsystem $\{i\}$ and the subsystem $\{j, k\}$. Each Hilbert direction-subspace has a dimension growing with the number of time steps, so we use the generalization of the negativity for higher-dimensional systems (so as to have $0 \leqslant N \leqslant 1$ ) [30]. We have calculated $N^{(3)}$ in $\mathrm{AQW}_{(3)}$, with the walker starting at the origin and initial coin state $\operatorname{col}(1, i) / \sqrt{2}$, for a number of time steps $t$ up to 10 , obtaining the plot in Fig. 2(e). Even if the number of time steps considered here is not so large (due to the dimension of the total Hilbert space, the computational power required for the evaluation grows rapidly with $t$ ), it is easy to check that AQW (3) is able to generate a considerable amount of genuine multipartite entanglement. It is also interesting to notice that $N^{(3)}$ saturates rather fast.

\section{IMPLEMENTABILITY OF $N$-DIMENSIONAL ALTERNATE QUANTUM WALK}

Let us finally discuss the implementability of $\mathrm{AQW}_{(N)}$. Realizing $\mathrm{QW}_{(N)}$ is quite demanding because of the complexity of performing coin operators to transform the needed $2^{N}$-dimensional qudit. On the other hand, in $\mathrm{AQW}_{(N)}$ : (i) a single coin-qubit is required independently of $N$; (ii) twodimensional transformations of the qubit are easy to implement [5-7]; and (iii) the sequential application of operators $\hat{D}_{j} \hat{C}_{j}$, $j=1, \ldots, N$, makes the implementation of $\mathrm{AQW}_{(N)}$ similar to that of $\mathrm{QW}_{(1)}$, provided that all $N$ dimensions could be multiplexed into a single one (similarly to what Schreiber et al. [12] have recently done in their pioneering implementation of $\left.\mathrm{QW}_{(2)}\right)$. Indeed, one could even implement $\mathrm{AQW}_{(N)}$ with a constant number of physical elements independently of $N$ if there is sufficient control on the experimental device.

In order to illustrate this, we generalize the idealized device already discussed in Refs. [20,31], which is similar to that actually used in Ref. [5]. Consider a long enough optical cavity containing two electro-optic modulators (EOMs) whose roles are (i) EOM1 performs the coin operator $\hat{C}$ (i.e., makes a unitary transformation of the light polarization state, which plays the role of the coin qubit in this implementation of the walk); and (ii) EOM2 performs the conditional displacement $\hat{D}$, which consists in up/down shifting the carrier frequency of the light pulse depending on its polarization. The light pulses entering the cavity are assumed to be much shorter than the cavity length, and the frequency shifts introduced by EOM 2 must be large enough for avoiding any frequency overlapping between pulses. With such a device not only $\mathrm{QW}_{(1)}$ can be implemented-see Refs. [5,20,31]—but also AQW $(N)$ could be implemented for different values of $N$ by properly programming the operations of the EOMs, without the need for additional elements. For example, in order to perform $\mathrm{AQW}_{(2)}$, the first half of the time step is implemented within one cavity round trip of the light pulse (during which EOM1 and EOM2 implement $\hat{D}_{1} \hat{C}_{1}$ ); then, in the subsequent cavity round trip, the settings of both EOMs are changed in order to perform a different coin operator and a different frequency displacement implementing $\hat{D}_{2} \hat{C}_{2}$. Importantly, the frequency displacements in $\hat{C}_{1}$ and $\hat{C}_{2}$ must be different enough in order to multiplex a large number of steps [12]. Only technical limitations seem to restrain the extension of the procedure to higher $N$ [32]. However, we are not claiming that the device just outlined is the most appropriate for implementing AQW $\mathrm{A}_{(N)}$. Indeed, a suitable modification of the flexible device used by Schreiber et al. [7,12] would probably be a more promising option. With our discussion we just want to emphasize that a single and conceptually simple device could implement alternate quantum walks with tunable dimensionality.

\section{CONCLUSIONS}

In conclusion, we have introduced the $N$-dimensional alternate quantum walk and discussed some of its properties through the analysis of the dispersion relation that reveals, in particular, the existence of diabolical points. We have demonstrated that for $N=3$, the process generates genuine multipartite entanglement. We have finally discussed its implementability, that could be possible with physical resources that do not necessarily grow with the dimensionality of the walk.

\section{ACKNOWLEDGMENTS}

We thank Th. Busch, M. Mc Gettrick, C. M. Chandrashekar, C. Navarrete-Benlloch, A. Pérez, A. Romanelli, and M. Hinarejos for discussions. This work has been supported by the Spanish Government and the European Union FEDER through Projects No. FIS2008-06024-C03-01 and No. FIS2011-26960, by the Basque Government through Grant No. IT472-10, and by the UK EPSRC, Grant No. EP/G004579/1, under the "New directions for EPSRC research leaders" initiative. C.D.F. acknowledges A. Pérez and the Universitat de València for the kind hospitality.
[1] Y. Aharonov, L. Davidovich, and N. Zagury, Phys. Rev. A 48, 1687 (1993); D. Meyer, J. Stat. Phys. 85, 551 (1996); J. Watrous, Proceedings of the 33rd Annual ACM Symposium on the Theory of Computing, Heraklion, Crete,
Greece, July 06-08, 2001 (ACM Press, New York, 2001), p. 60.

[2] E. Farhi and S. Gutmann, Phys. Rev. A 58, 915 (1998); A. M. Childs and J. Goldstone, ibid. 70, 042312 (2004). 
[3] F. W. Strauch, Phys. Rev. A 73, 054302 (2006).

[4] A. Ambainis, Int. J. Quant. Inf. 1, 507 (2003); J. Kempe, Contemp. Phys. 44, 307 (2003); V. Kendon, Math. Struct. Comp. Sci. 17, 1169 (2006); Philos. Trans. R. Soc., A 364, 3407 (2006); N. Konno, in Quantum Walks, edited by U. Franz and M. Schürmann, Quantum Potential Theory, Lecture Notes in Mathematics Vol. 1954 (Springer, Berlin, 2008).

[5] D. Bouwmeester, I. Marzoli, G. P. Karman, W. Schleich, and J. P. Woerdman, Phys. Rev. A 61, 013410 (1999).

[6] B. Do et al., J. Opt. Soc. Am. B 22, 499 (2005); C. A. Ryan, M. Laforest, J. C. Boileau, and R. Laflamme, Phys. Rev. A 72, 062317 (2005); P. Zhang, X. F. Ren, X. B. Zou, B. H. Liu, Y. F. Huang, and G. C. Guo, ibid. 75, 052310 (2007); P. H. Souto Ribeiro, S. P. Walborn, C. Raitz, L. Davidovich, and N. Zagury, ibid. 78, 012326 (2008); M. Karski et al., Science 325, 174 (2009); H. B. Perets, Y. Lahini, F. Pozzi, M. Sorel, R. Morandotti, and Y. Silberberg, Phys. Rev. Lett. 100, 170506 (2008); H. Schmitz, R. Matjeschk, C. Schneider, J. Glueckert, M. Enderlein, T. Huber, and T. Schaetz, ibid. 103, 090504 (2009); F. Zähringer, G. Kirchmair, R. Gerritsma, E. Solano, R. Blatt, and C. F. Roos, ibid. 104, 100503 (2010); M. A. Broome, A. Fedrizzi, B. P. Lanyon, I. Kassal, A. Aspuru-Guzik, and A. G. White, ibid. 104, 153602 (2010).

[7] A. Schreiber, K. N. Cassemiro, V. Potocek, A. Gabris, P. J. Mosley, E. Andersson, I. Jex, and C. Silberhorn, Phys. Rev. Lett. 104, 050502 (2010); A. Schreiber, K. N. Cassemiro, V. Potocek, A. Gabris, I. Jex, and C. Silberhorn, ibid. 106, 180403 (2011).

[8] A. Peruzzo et al., Science 329, 1500 (2010); J. C. F. Matthews et al., arXiv:1106.1166 [quant-ph]; J. O. Owens et al., New J. Phys. 13, 075003 (2011); L. Sansoni, F. Sciarrino, G. Vallone, P. Mataloni, A. Crespi, R. Ramponi, and R. Osellame, Phys. Rev. Lett. 108, 010502 (2012).

[9] T. D. Mackay, S. D. Bartlett, L. T. Stephenson, and B. C. Sanders, J. Phys. A: Math. Gen. 35, 2745 (2002).

[10] C. Moore and A. Russell, in Proceedings of RANDOM, 2002, edited by J. D. P. Rolim and P. Vadham (Springer, Cambridge, MA, 2002), pp. 164-178.

[11] E. Roldán and J. C. Soriano, J. Mod. Opt. 52, 2649 (2005).

[12] A. Schreiber et al., Science 336, 55 (2012).

[13] B. Tregenna et al., New. J. Phys. 5, 83 (2003); I. Carneiro et al., ibid. 7, 156 (2005); A. C. Oliveira, R. Portugal, and R. Donangelo, Phys. Rev. A 74, 012312 (2006); K. Watabe,
N. Kobayashi, M. Katori, and N. Konno, ibid. 77, 062331 (2008); M. Stefanák et al., Phys. Scr., T 140, 014035 (2010); N. Shenvi, J. Kempe, and K. BirgittaWhaley, Phys. Rev. A 67, 052307 (2003).

[14] N. Inui, Y. Konishi, and N. Konno, Phys. Rev. A 69, 052323 (2004).

[15] C. Di Franco, M. Mc Gettrick, and Th. Busch, Phys. Rev. Lett. 106, 080502 (2011).

[16] C. Di Franco, M. Mc Gettrick, T. Machida, and Th. Busch, Phys. Rev. A 84, 042337 (2011).

[17] M. V. Berry and M. Wilkinson, Proc. R. Soc. A 392, 15 (1984).

[18] M. V. Berry and M. R. Jeffrey, Prog. Opt. 50, 13 (2007).

[19] G. J. de Valcárcel, E. Roldán, and A. Romanelli, New J. Phys. 12, 123022 (2010).

[20] P. L. Knight, E. Roldán, and J. E. Sipe, Phys. Rev. A 68, 020301 (2003); J. Mod. Opt. 51, 1761 (2006).

[21] G. J. de Valcárcel, M. Hinarejos, E. Roldán, A. Pérez, and A. Romanelli, arXiv:1212.3600 [quant-ph].

[22] C. A. Mead and D. G. Truhlar, J. Chem. Phys. 70, 2284 (1979); L. S. Cederbaum, R. S. Friedman, V. M. Ryaboy, and N. Moiseyev, Phys. Rev. Lett. 90, 013001 (2003).

[23] A. Bostwick et al., New J. Phys. 9, 385 (2007).

[24] A. H. Castro Neto et al., Rev. Mod. Phys. 81, 109 (2009).

[25] M. Zhang, H. H. Hung, Ch. Zhang, and C. Wu, Phys. Rev. A 83, 023615 (2011).

[26] S. M. Young, S. Zaheer, J. C. Y. Teo, C. L. Kane, E. J. Mele, and A. M. Rappe, Phys. Rev. Lett. 108, 140405 (2012).

[27] Y. Omar, N. Paunković, L. Sheridan, and S. Bose, Phys. Rev. A 74, 042304 (2006); S. E. Venegas-Andraca and S. Bose, arXiv:0901.3946 [quant-ph]; P. Xue and B. C. Sanders, ibid. 85, 022307 (2012).

[28] R. Horodecki et al., Rev. Mod. Phys. 81, 865 (2009).

[29] C. Sabin and G. Garcia-Alcaine, Eur. Phys. J. D 48, 435 (2008).

[30] S. Lee, D. P. Chi, S. D. Oh, and J. Kim, Phys. Rev. A 68, 062304 (2003).

[31] P. L. Knight, J. E. Sipe, and E. Roldán, Opt. Commun. 227, 147 (2003).

[32] The multiplexing requires that the frequency displacements corresponding to different dimensions have to be very different. (In fact, this difference determines the maximum number of steps that can be implemented; see the discussion in Ref. [12]). Moreover, the frequency shifts should be done with a high repetition rate (larger than the inverse of the cavity round-trip time). All this could be very demanding for actual EOMs. 